\title{
Strange Messages: Chemical and Thermal Freeze-out in Nuclear Collisions
}

\author{
U Heinz \\ CERN/TH, CH-1211 Geneva 23, Switzerland; and Institut für Theoretische Physik, \\ Universität Regensburg, D-93040 Regensburg, Germany
}

\begin{abstract}
Thermal models are commonly used to interpret heavy-ion data on particle yields and spectra and to extract the conditions of chemical and thermal freezeout in heavy-ion collisions. I discuss the usefulness and limitations of such thermal model analyses and review the experimental and theoretical evidence for thermalization in nuclear collisions. The crucial role of correlating strangeness production data with single particle spectra and two-particle correlation measurements is pointed out. A consistent dynamical picture for the heavy-ion data from the CERN SPS involves an initial prehadronic stage with deconfined color and with an appreciable isotropic pressure component. This requires an early onset of thermalization.

PACS numbers: 24.10.Pa, 25.75.-q, 25.75.Dw, 25.75.Ld
\end{abstract}

\section{Introduction}

The stated goal of the relativistic heavy-ion programs at CERN and BNL is the study of the phase diagram of strongly interacting matter at high temperatures and densities and the search for the quark-gluon plasma (QGP). The discussion of a phase diagram requires thermodynamic language. A phase transition from an initial color-deconfined QGP to a color-confined hadronic state (as it is supposed to occur in heavy-ion collisions) can only be reasonably well defined if the system under study is in a state of approximate local thermodynamic equilibrium. The application of thermal and hydrodynamic models to relativistic heavy-ion data is therefore more than a poor man's approach to heavy-ion dynamics, it is rather a necessity for everybody who wants to convince himself and others that we succeeded in creating the quark-gluon plasma (QGP) and observing the phase transition accompanying its hadronization.

Of course, the models may fail; in fact, they must necessarily fail beyond a certain level of detail when applied to heavy-ion data. The reason is obvious: the collision systems are small, causing corrections to the infinite volume limit usually assumed in the thermodynamic approach, and they undergo a strong dynamical evolution on time scales which are comparable to the microscopic thermalization time. Thermal models therefore can never provide more than a rough picture of the bulk of the phenomena, good for qualitative answers; on a more detailed and quantitative level, the failure 
of the thermal model will become manifest, and traces of genuine QCD dynamics (as opposed to thermodynamics) will show up. But when trying to assess bulk phenomena like QGP formation, we are not (in fact, we must not be) primarily interested in these deviations from thermodynamic behaviour and the traces of elementary QCD dynamics; the latter can be studied much more easily and cleanly in elementary lepton or hadron collisions. We should rather concentrate on the rough global pattern of the data and try to understand them within a (nota bene: sufficiently sophisticated, see below) thermoand hydrodynamic approach. On the other hand, if it turns out that not even the rough qualitative features of the data can be understood in this way and detailed hadronic dynamics is required even for a superficial understanding of the observations, then we should concede that our attempt to create "hot QCD matter" has failed.

Having argued in favor of a "simple" thermal approach to heavy-ion data, the next questions to be addressed are (i) the level of sophistication which the thermal model should have before being applicable to the description of particle production in nuclear collisions, (ii) which level of agreement between model and data can at best be expected, and (iii) where to draw the line between agreement and disagreement when comparing model and data. This is what this contribution is about. At the end I will try to draw some conclusions about what we have learnt so far from the thermal analysis of heavy-ion data, and which further steps should be taken.

\section{Two types of "thermal" behaviour}

"Thermal" behaviour can arise in conceptually different ways, with different meanings of the "temperature" parameter $T$. For us the two most important variants of "thermal" behaviour are the following:

1. The statistical occupation of hadronic phase-space with minimum information. The latter is in practice provided by external constraints on the total available energy $E$, baryon number $B$, strangeness $S$ and, possibly, a constraint $\lambda_{s}$ on the overall fraction of strange hadrons. "Thermal" behaviour arises in this case via the Maximum Entropy Principle in which the "temperature" $T$ and "fugacities" $e^{\mu_{b} / T}, e^{\mu_{s} / T}$ (which in the canonical approach are replaced by so-called "chemical factors" [1, 2]) occur as Lagrange multipliers for the constraints. Examples are nucleon emission from an evaporating compound nucleus in low-energy nuclear physics and hadronization in $e^{+} e^{-}, p p$ and $p \bar{p}$ collisions (hadron yields [1, 2] and $m_{\perp}$-spectra [3]). The number of parameters to fit the data in such a situation is equal to the number of "conserved quantities" (constraints), and it reflects directly the information content of the fitted observable(s). This type of "thermal" behaviour requires no rescattering and no interactions among the hadrons, there is no isotropic pressure and no collective flow in the hadronic final state and, in fact, the concept of local equilibrium can not be applied. Of course, this type of "thermal"

behaviour is not really what we are interested in in heavy ion collisions, except as a baseline against which to differentiate interesting phenomena. 
2. Thermalization of a non-equilibrium initial state by kinetic equilibration (rescattering). This does require (strong!) interactions among the hadrons. Here one must differentiate between thermal equilibration (reflected in the shape of the momentum spectra), which defines the temperature $T$, and chemical equilibration (reflected in the particle yields and ratios) which defines the chemical potentials in a grand canonical description. The first is driven by the total hadron-hadron cross section while the second relies on usually much smaller inelastic cross sections and thus happens more slowly. This type of equilibrium is accompanied by pressure which drives collective flow (radial expansion into the vacuum as well as directed and elliptic flow in non-central collisions). In heavy ion collisions it is realized at most locally, in the form of local thermal and/or chemical equilibrium - due to the absence of confining walls there is never a global equilibrium. This is the type of "thermal" behaviour which we are searching for in heavy-ion collisions.

I stress that flow is an unavoidable consequence of this type of equilibration. Thermal fits without flow to hadron spectra are not consistent with the kinetic thermalization hypothesis. Flow contains information; it is described by three additional fit parameters $\vec{v}(x)$. This information is related to the pressure history in the early stages of the collision and thereby (somewhat indirectly) to the equation of state of the hot matter.

Most thermal fits work with global parameters $T$ and $\mu$ which, at first sight, appears inconsistent with what I just said. Here the role of freeze-out becomes important: freezeout cuts off the hydrodynamical evolution of the thermalized region via a kinetic freezeout criterium [4] which involves the particle densities, cross sections and the expansion rate. In practice freeze-out may, but need not occur at nearly the same temperature everywhere [4].

Clearly a thermal fit to hadron production data (if it works) is not the end, but rather the beginning of our understanding. One must still check the dynamical consistency of the fit parameters $T_{f}, \mu_{f}, \vec{v}_{f}$ : can one find equations of state and initial conditions which yield such freeze-out parameters? Which dynamical models can be excluded?

\section{The hadronic phase diagram}

In figure 11 I show a recent version of the phase diagram for strongly interacting matter, with various sets of data points included [5, 6, 7, 8, 9]. In the present section I discuss the meaning of this figure and explain how these data points were obtained. In the next section I will discuss some problems connected with the extraction procedures.

\subsection{Chemical freeze-out}

3.1.1. $e^{+} e^{-}$and $p p$ collisions. Let me begin with the $e^{+} e^{-}$data point in figure 1 . (There is also a $p p$ point from Ref. [2] which was omitted for clarity.) In spite of 
Figure 1. Compilation of freezeout points from SIS to SPS energies. Filled symbols: chemical freeze-out points from hadron abundances. Open symbols: thermal freezeout points from momentum spectra and two-particle correlations. For each system, chemical and thermal freeze-out were assumed to occur at the same value $\mu_{B} / T$. The shaded region indicates the parameter range of the expected transition to a QGP.

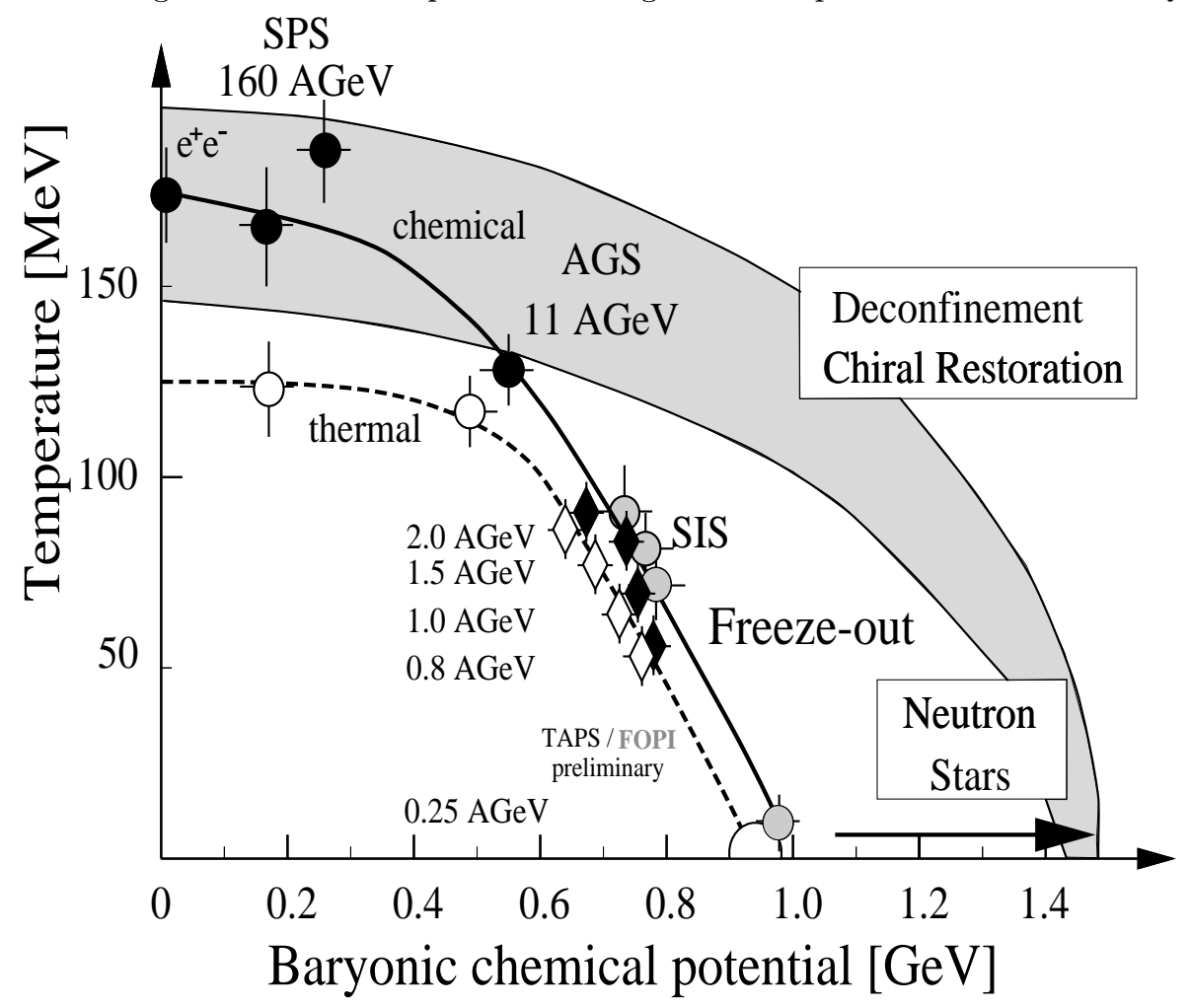

what I said about case 1. above, a "thermal" analysis of hadron yields in elementary collisions [1, 2] is still interesting. (Of course, in this case the canonical formalism must be used, due to the small collision volume.) The interest arises a posteriori from the observed universality of the fit parameters, namely a universal "hadronization" or "chemical freeze-out" temperature $T_{\text {chem }}=T_{\text {had }} \approx 170 \mathrm{MeV}$ (numerically equal to the old Hagedorn temperature $T_{\mathrm{H}}$ and consistent with the inverse slope parameter of the $m_{T}$-spectra in $p p$ collisions [3]), and a universal strangeness fraction $\lambda_{s}=$ $2\langle\bar{s} s\rangle /\left.(\langle\bar{u} u\rangle+\langle\bar{d} d\rangle)\right|_{\text {produced }} \approx 0.2-0.25$, almost independent of $\sqrt{s}$ 1, 2, 10].

This is most easily understood [2] in terms of a universal critical energy density $\epsilon_{\text {crit }}$ for hadronization which, via the Maximum Entropy Principle, is parametrized by a universal "hadronization temperature" $T_{\text {had }}$ and which, according to Hagedorn, characterizes the upper limit of hadronic phase-space. Supporting evidence comes from the observed increase with $\sqrt{s}$ of the fitted fireball volume $V_{f}$ (which accomodates the increasing multiplicities and widths of the rapidity distributions). Although higher collision energies result in larger initial energy densities $\epsilon_{0}$, the collision zone subsequently undergoes more (mostly longitudinal and not necessarily hydrodynamical) expansion until $\epsilon_{\text {crit }}$ is reached and hadron formation can proceed. The systematics of the data can only be understood if hadron formation at $\epsilon>\epsilon_{\text {crit }}$ (i.e. $T>T_{\mathrm{H}}$ for 
the corresponding Lagrange multipliers) is impossible. With this interpretation, the chemical analysis of $e^{+} e^{-}, p p$ and $p \bar{p}$ collisions does provide one point in the $T-\mu_{b}$ phase diagram (see figure 1). - The only "childhood memory" of the collision system is reflected in the low value of $\lambda_{s}$, indicating suppressed strange quark production (relative to $u$ and $d$ quarks) in the early pre-hadronic stages of the collision.

3.1.2. AA collisions: strangeness enhancement. In this light the observation [5] of a chemical freeze-out temperature $T_{\text {chem }} \approx T_{\mathrm{H}} \approx 170 \mathrm{MeV}$ in heavy-ion collisions with sulphur beams at the SPS (figure 1), taken by itself, is not really interesting. It suggests that in heavy-ion collisions hadronization occurs via the same statistical hadronic phasespace occupation process as in $p p$ collisions. What is interesting, however, is the observation [10, 11] that the global strangeness fraction $\lambda_{s} \approx 0.4-0.45$ in $A A$ collisions is about a factor 2 larger than in $e^{+} e^{-}$and $p p$ collisions. If $p p$ and $\mathrm{S}+A$ collisions hadronize via the same mechanism, and in $\mathrm{S}+A$ collisions the Maximum Entropy particle yields fixed at $T_{\text {had }}$ are not modified by inelastic hadronic final state rescattering, this increase in $\lambda_{s}$ must reflect a difference in the properties of the prehadronic state! In nuclear collisions the prehadronic stage allows for more strangeness production, most likely due to a longer lifetime before hadronization.

It was noted before [10, 11] that the global strangeness enhancement occurs already in collisions between medium size nuclei $(\mathrm{S}+\mathrm{S})$ and remains roughly unchanged in $\mathrm{Pb}+\mathrm{Pb}$ collisions. In this conference we saw data from the WA97 collaboration 12, 13, 14 which provide two important further details:

1. While the bulk of the strangeness enhancement from $p+\mathrm{Pb}$ to $\mathrm{Pb}+\mathrm{Pb}$ collisions is carried by the kaons and hyperons $(\Lambda, \Sigma)$, which are enhanced by about a factor 3 near midrapidity, the enhancement is much stronger for the doubly and triply strange baryons $\Xi$ and $\Omega$ and their antiparticles, with an enhancement factor of about 17 (!) for $\Omega+\bar{\Omega}$ at midrapidity. The enhancement clearly scales with the strangeness content [13], as naively expected in statistical and thermal models, but in stark contradiction to expectations based on the consideration of the respective production thresholds in hadronic (re)interactions.

2. In semicentral $\mathrm{Pb}+\mathrm{Pb}$ collisions the enhancement grows linearly with the number of participating nucleons in the collision, so the enhanced yield of all measured strange hadron species per participating nucleon is independent of the effective size of the colliding system from about 150 to 400 participants [12]. Where comparison is possible, this systematics even carries over to central $\mathrm{S}+\mathrm{S}$ collisions [14 with as few as 55-60 participating nucleons. So whatever causes the enhancement in $\mathrm{Pb}+\mathrm{Pb}$ collisions (e.g. the existence of a color-deconfined prehadronic stage) is not particular to (semi)central $\mathrm{Pb}+\mathrm{Pb}$ collisions, but exists already in $\mathrm{S}+\mathrm{S}$ collisions! I will return to the $A$ independence of this effect below.

At most half of the $100 \%$ increase of global strangeness production in $A A$ collisions can be explained 15] by the removal of canonical constraints in the small $e^{+} e^{-}$and $p p$ collision volumes (which would be an interesting observation in itself because it 
would already imply that in nuclear collisions hadron production and the conservation of quantum numbers occurs no longer on nucleonic, but indeed on nuclear length scales). The remainder of the increase must be due to extra strangeness production in the whole fireball volume before hadronization. It is interesting to analyze in the same way the strong $\Omega+\bar{\Omega}$ enhancement in $\mathrm{Pb}+\mathrm{Pb}$ (by a factor 17 relative to $p+\mathrm{Pb}[13]$ ): of course, the $\Omega$ (carrying 3 units of strangeness) suffers a particularly strong canonical suppression due to exact strangeness conservation in the small hadronization volume of a $p p$ (or $p+\mathrm{Pb}$ ) collision; for $T_{\text {had }} \simeq 170 \mathrm{MeV}, \gamma_{\mathrm{s}}=0.5$ and $V=17.6 \mathrm{fm}^{3}$ (as obtained by fitting $p p$ data at $\sqrt{s}=27 \mathrm{GeV}$ [2] $\Omega+\bar{\Omega}$ are canonically suppressed by a factor 12 relative to a grand canonical treatment [16]. Again the observed enhancement effect in $\mathrm{Pb}+\mathrm{Pb}$ collisions is considerably larger than expected from a simple removal of the canonical constraints.

The observed strangeness fraction $\lambda_{s}=0.45$ in nuclear collisions corresponds to a strangeness saturation coefficient [17] $\gamma_{s} \approx 0.7$ [10]. On the other hand, a value of $\gamma_{s} \approx 0.7$ in the hadronic final state may, in fact, be the upper limit reachable in heavy-ion collisions 15 because the corresponding strangeness fraction agrees with that in a fully equilibrated QGP at $T_{\text {had }} \approx 170 \mathrm{MeV}$. If both strangeness and entropy are conserved or increase similarly during hadronization, $\gamma_{s} \approx 0.7$ in the Maximum Entropy particle yield after hadronization would be a universal consequence of a fully thermally and chemically equilibrated QGP before hadronization [15]. The SPS data would then be completely consistent with such a prehadronic state.

The existence of a prehadronic stage without color confinement, both in $\mathrm{S}+\mathrm{S}$ and $\mathrm{Pb}+\mathrm{Pb}$ collisions at the SPS, is also suggested by an analysis of baryon/antibaryon ratios of different strangeness. This was stressed at this conference by A. Bialas [18] who redid this analysis with the new data following the ideas of Rafelski [17].

According to figure 1 chemical freeze-out in sulphur-induced collisions at the SPS appears to occur right at the critical line, i.e. immediately after hadronization. The SIS data, on the other hand, indicate much lower chemical freeze-out temperatures. The origin of this is probably due to longer lifetimes of the reaction zone at lower beam energies, allowing for some chemical re-equilibration by inelastic hadronic reactions.

A tendency for some chemical re-equilibration after the hadronization of the proposed pre-hadronic stage may also be visible in the still preliminary $\mathrm{Pb}+\mathrm{Pb}$ data at the SPS: although thermal model analyses of these data still give wildly scattering results [10, 7, 19, some authors [19] find chemical freeze-out temperatures in $\mathrm{Pb}+\mathrm{Pb}$ below $140 \mathrm{MeV}$. A thermal model analysis of RQMD simulations also gives chemical freeze-out temperatures of $172 \mathrm{MeV}$ in $\mathrm{S}+\mathrm{S}$, but of only $155 \mathrm{MeV}$ in $\mathrm{Pb}+\mathrm{Pb}$ collisions [20]. Both analyses show a characteristic failure to reproduce the $\Omega$ and $\bar{\Omega}$ yields 12 . This was interpreted [20, 21] in terms of early freeze-out of these triply strange baryons due to their small interaction cross sections with other types of hadrons. It is interesting to observe that in the thermal analysis of the data [19] the model underpredicts the measured $\Omega$ and $\bar{\Omega}$ yields (which prefer a higher freeze-out temperature $T \geq 170 \mathrm{MeV}$ [7, 10]) while in RQMD, which is known to produce too few $\Omega$ and $\bar{\Omega}$ baryons in $p p$ and 
$p A$ collisions, the thermal model overpredicts the simulated yields. All this points to the $\Omega$ and $\bar{\Omega}$ as relatively early hadronic messengers, and the message they seem to carry in the $\mathrm{Pb}+\mathrm{Pb}$ data is again that of the existence of a prehadronic stage with enhanced global strangeness which hadronizes statistically at $T_{\text {had }}=T_{\mathrm{H}} \simeq 170 \mathrm{MeV}$.

\subsection{Flow and thermal freeze-out}

The other important observation in the hadronic sector of nuclear collisions is that of collective flow (radial expansion flow, directed and elliptical flow). It is usually extracted from the shape of the single-particle momentum distributions. Radial flow, for example, leads to a flattening of the $m_{\perp}$-spectra. For the analysis one must distinguish two domains. In the relativistic domain $p_{\perp} \gg m_{0}$ the inverse slope $T_{\text {app }}$ of all particle species is the same and given by the blueshift formula $4 \pi T_{\text {app }}=T_{f} \sqrt{\left(1+\left\langle v_{\perp}\right\rangle\right) /\left(1-\left\langle v_{\perp}\right\rangle\right)}$. This formula does not allow to disentangle the average radial flow velocity $\left\langle v_{\perp}\right\rangle$ and freezeout temperature $T_{f}$. In the non-relativistic domain $p_{\perp} \ll m_{0}$ the inverse slope is given approximately by $T_{\mathrm{app}}=T_{f}+m_{0}\left\langle v_{\perp}^{2}\right\rangle$, and the rest mass dependence of the "apparent temperature" (inverse slope) allows to determine $T_{f}$ and $\left\langle v_{\perp}^{2}\right\rangle$ separately. (In $p p$ collisions no $m_{0}$-dependence of $T_{\text {app }}$ is seen [22].) Plots of $T_{\text {app }}$ against $m_{0}$ were shown in several talks at this conference, showing that the data follow very nicely this systematics, from SIS to SPS energies.

A notable exception are the $\Omega$-spectra of WA97 [12] which are steeper than expected from this formula. Again, as in the above discussion of their abundance, this reflects their character as "early hadronic messengers" [21]: the $\Omega$ and $\bar{\Omega}$ are the only baryons which (due to quantum number mismatch) do not have a strong resonance with pions, the most abundant particles in the fireball. Since resonance scattering is the most efficient thermalization mechanism in a dense hadronic system, the $\Omega$ and $\bar{\Omega}$ momentum distributions freeze out earlier than those of all other baryons. This implies [21] that they cannot efficiently pick up the collective transverse flow which builds up among the pions in the later stages of the expansion, and their spectra reflect the much weaker collective transverse flow in the early collision stages, just after hadron formation.

[This also illustrates the important role of the baryon contamination in the hot fireball: in $\mathrm{Pb}+\mathrm{Pb}$ collisions at the SPS the pion and baryon spectra decouple late and cool down to rather low temperatures of about $120 \mathrm{MeV}$ (see below) because the pions are "glued" together by the baryons via resonance scattering. At RHIC this glue will be less efficient since near midrapidity there will essentially exist only baryon-antibaryon pairs at rather low thermal equilibrium abundances. It is thus expected that at RHIC thermal decoupling occurs at somewhat higher freeze-out temperatures, closer to the hadronization phase transition.]

The separation of collective flow and random thermal motion from an analysis of single particle spectra is not uncontroversial. The main reason is that the fitted values for $T$ and $v_{\perp}$ tend to be strongly correlated. To break the correlation one must study spectra of hadrons with different masses in the low- $p_{\perp}$ region which, on the other hand, 
Figure 2. Thermal freeze-out temperature and transverse flow velocities extracted from fits to the transverse momentum spectra of negative hadrons $\left(h^{-}\right)$and deuterons (d) and to the transverse HBT radius $(2 \pi-\mathrm{BE})$. The shaded area indicates the overlap region near $T_{\text {f.o. }} \approx 120 \mathrm{MeV}$ and $v_{\perp} \approx 0.55 \mathrm{c}$. (From Ref. 24].)

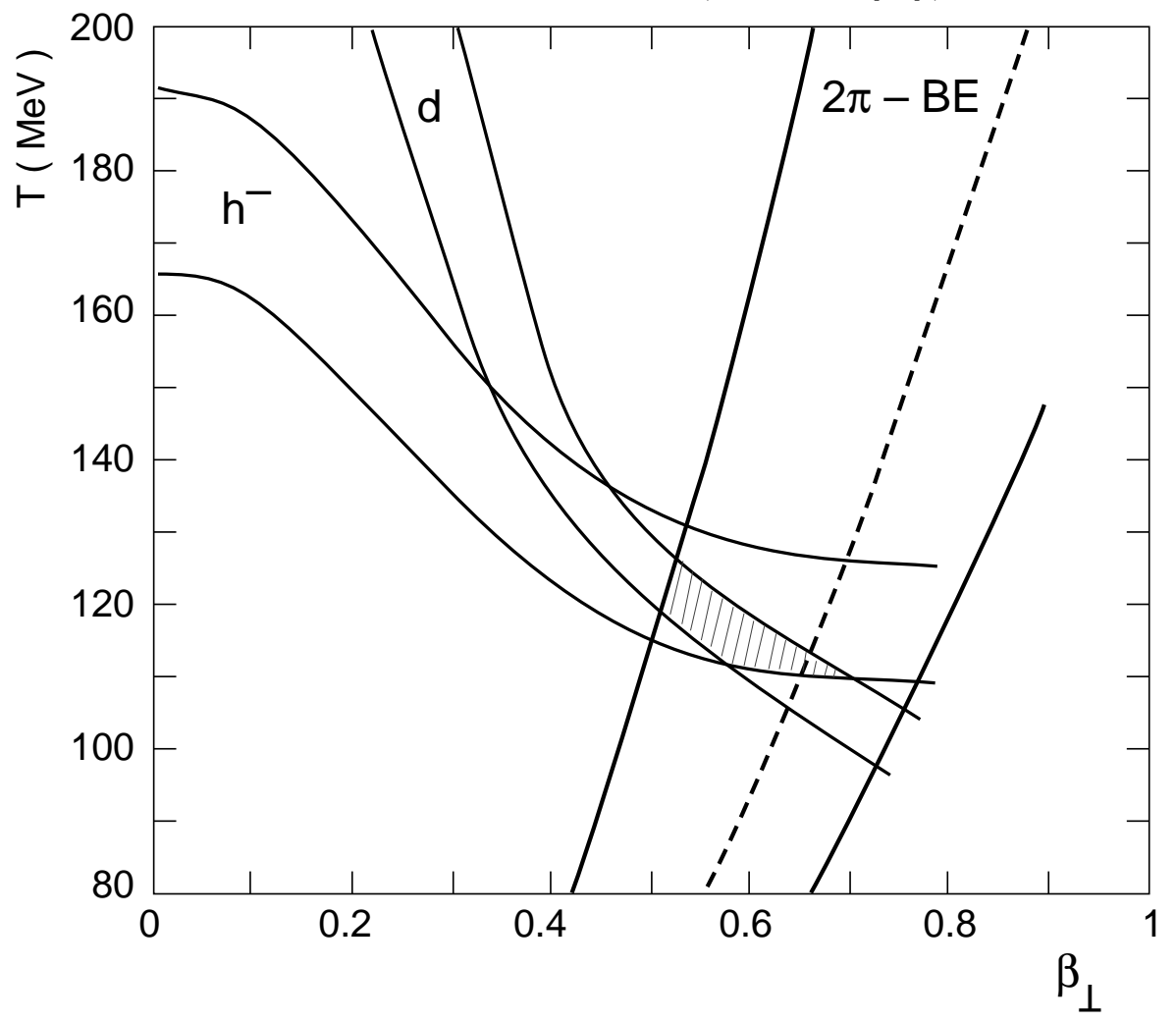

is contaminated by post-freeze-out resonance decays. As a consequence, fits done in different $p_{\perp}$ windows tend to give different results.

A clearer determination of the transverse collective flow comes from a direct measurement of the flow-induced space-momentum correlations via the $M_{\perp}$-dependence of the two-pion HBT radii [23]. As shown in [24, 25] the correlations between temperature and transverse flow in a fit of the single particle transverse momentum spectra and of the transverse two-particle HBT radii are essentially orthogonal to each other (see figure 2), and the combined analysis of spectra and correlations allows for a clean separation of random thermal motion from collective flow.

This analysis [24, 25] gave rise to the open circle for the SPS data in figure 1, indicating the point of thermal decoupling in $158 \mathrm{~A} \mathrm{GeV} / c \mathrm{~Pb}+\mathrm{Pb}$ collisions $\left(T_{\text {therm }} \approx 120 \mathrm{MeV},\left\langle v_{\perp}\right\rangle \approx 0.5 c\right)$. It is consistent with a comparison of the spectral slopes for different mass hadrons by Kämpfer [26]. Earlier analyses of S+S data [27] showed a thermal decoupling at $T_{\text {therm }} \approx 140-150 \mathrm{MeV},\left\langle v_{\perp}\right\rangle \approx 0.25-0.35 c$. The open circle for the AGS as well as the open quadrangles for the SIS in figure 1 were obtained similarly as in [26, 27] by comparing transverse momentum spectra of particles with different masses (see [7, 8] for references). All open symbols correspond to heavy collision systems $(\mathrm{Pb}+\mathrm{Pb}, \mathrm{Au}+\mathrm{Au})$. The dashed line connects them by eye in an attempt to 
construct a "thermal freeze-out curve" for heavy-ion collisions of size $200+200$.

It is interesting to analyze the $A$-dependence of the various hadronic observables, i.e. of strangeness enhancement, chemical and thermal freeze-out temperatures and radial transverse flow. This discussion should include the available information on the impact parameter dependence in $\mathrm{Pb}+\mathrm{Pb}$ collisions since collisions at different impact parameters also involve different numbers $N_{\text {part }}$ of participating nucleons. Whereas the freeze-out temperatures (certainly the thermal freeze-out temperature, but perhaps also the chemical one, see above) seem to come down with increasing size of the collision system, while at the same time the strength of the radial transverse flow goes up, the strangeness enhancement (i.e the strange particle production per participating nucleon relative to $p p$ and $p A$ collisions) appears to be independent of the number of $N_{\text {part }}$, at least in the range $60 \leq N_{\text {part }} \leq 400$. The buildup of radial flow is largely (although not exclusively) a hadronic reinteraction phenomenon [21]; the same is true for the freezeout process. The $N_{\text {part }}$-dependence of both features can be explained in terms of the longer lifetime of the reaction zone as $N_{\text {part }}$ increases, giving the system more time to re-equilibrate, cool down and develop collectivity.

In contrast, the $A$-independence of the strangeness enhancement features suggest that they are not due to hadronic re-interactions, but originate in a prehadronic phase with properties which are essentially independent of the system size once $N_{\text {part }} \geq 50$ or so. My interpretation of these facts is that at SPS energies the energy density threshold for QGP has been overcome by a sizeable margin [28], and that even in small collision systems a deconfined phase is created which interacts sufficiently long and sufficiently strongly to approximately saturate strangeness production. One can even argue that isotropic pressure (a signature of local thermalization) must be present at this early stage [28], and that the observed elliptic flow in non-central $\mathrm{Pb}+\mathrm{Pb}$ collisions [29] actually signals this early pressure [30. A future systematic investigation also of the range $1<N_{\text {part }}<100$ would be very useful to study the onset and saturation of thermal and collective behaviour as the size and lifetime of the collision system increases. It will not, however, be an efficient method to study the onset of deconfinement - for that one must go to lower beam energies.

\section{Limitations of thermal model analyses}

After having explained how the data points in figure 1 were obtained, I would now like to ask the notorious David Mermin question [31] "What's wrong with this phase diagram?" In other words, I want to point out in more detail certain unavoidable problems with thermal model analyses of heavy-ion data. Only by remaining conscious of the limitations of the thermal approach and avoiding the overinterpretation of uncontrollable details one can fully exploit its power in providing essential qualitative insight into the physics of heavy-ion collisions. 


\subsection{Rapidity dependence of particle ratios}

The first problem is of purely practical nature: no heavy-ion experiment so far has full $4 \pi$ acceptance for identified particles, and particle spectra are available in restricted windows of $p_{\perp}$ and $y$ only. The observed nearly exponential form of the $p_{\perp}$-spectra allows for an extrapolation of the yields to the full transverse momentum range without introducing large uncertainties (at least if the acceptance covers sufficiently low values of $\left.p_{\perp}\right)$. A similar extrapolation of the rapidity spectra is not possible: even in a system which is in perfect local thermal equilibrium, particles with different masses tend to have strongly different rapidity distributions. In practice these differences in the shape of the rapidity spectra are even stronger, in the sense that even particles and antiparticles (with obviously identical masses) have different rapidity distributions. Thus there is essentially no way of extrapolating the rapidity distributions without really measuring them.

That this is a serious problem for thermal model analyses is illustrated by the following example: consider a stationary, spherical fireball in global thermodynamic equilibrium. It emits hadrons with the following rapidity distributions:

$$
\frac{d N_{i}}{d y} \sim e^{-m_{i} \cosh y / T}\left[1+2 \frac{T}{m_{i} \cosh y}+2\left(\frac{T}{m_{i} \cosh y}\right)^{2}\right]
$$

These resemble Gaussians with widths $\Gamma_{i} \approx 2.35 \sqrt{\frac{T}{m_{i}}}$ (the approximation being valid for $\left.m_{i} \gg T\right)$. Clearly, the particle ratios $\left(d N_{i} / d y\right) /\left(d N_{j} / d y\right)$ then depend strongly on the position of the rapidity interval $d y$ : away from $y=0$ heavy particles will be much more strongly suppressed relative to light particles than near $y=0$. In this case a measurement of particle yields in a small rapidity window is completely useless for a thermal model analysis (irrespective of whether the window is located near $y=0$ or at $y \neq 0$ ) unless it is known a priori that the radiator is a stationary spherical fireball.

The presence of (strong) longitudinal flow in relativistic heavy-ion collisions does not help very much in this connection; only in the limit of infinite beam energy with exact longitudinal boost-invariance due to Bjorken scaling, resulting in flat rapidity distributions, is it possible to base a thermal analysis on data in a finite, narrow rapidity interval. At SPS energies and below, where Bjorken scaling is not observed, thermal fits of data in finite rapidity windows require that the thermal model yields are cut to the actual experimental acceptance; this induces serious dependences on the detailed model assumptions, for example about the strength and profile of the longitudinal and transverse flow of the source. This model-dependence was recently studied in some detail in Ref. 32.

Flow effects drop largely out, however, if one works with particle ratios obtained from $4 \pi$ yields. (Please note that this requires a measurement of some sort, not a blind extrapolation of data from a small window in $y$ and $p_{\perp}$ to full momentum space!) The insensitivity to hydrodynamic flow becomes exact if the freeze-out temperature and chemical potential is everywhere the same. If freeze-out occurs on a sharp hypersurface 
$\Sigma$, the total yield of particle species $i$ is then given by

$$
N_{i}=\int \frac{d^{3} p}{E} \int_{\Sigma} p^{\mu} d^{3} \sigma_{\mu}(x) f_{i}(x, p)=\int_{\Sigma} d^{3} \sigma_{\mu}(x) j_{i}^{\mu}(x)
$$

where

$$
j_{i}^{\mu}(x)=\int d^{4} p 2 \theta\left(p^{0}\right) \delta\left(p^{2}-m_{i}^{2}\right) p^{\mu} \frac{g_{i}}{e^{\left[p \cdot u(x)-\mu_{i}\right] / T} \pm 1}
$$

is the number current density of particle species $i$. In thermal equilibrium it is given by $j_{i}^{\mu}(x)=\rho_{i}(x) u^{\mu}(x)$ with

$$
\begin{aligned}
\rho_{i}(x) & =u_{\mu}(x) j_{i}^{\mu}(x)=\int d^{4} p 2 \theta\left(p^{0}\right) \delta\left(p^{2}-m_{i}^{2}\right) p \cdot u(x) f_{i}\left(p \cdot u(x) ; T, \mu_{i}\right) \\
& =\int d^{3} p^{\prime} f_{i}\left(E_{p^{\prime}} ; T, \mu_{i}\right)=\rho_{i}\left(T, \mu_{i}\right) .
\end{aligned}
$$

Here $E_{p^{\prime}}$ is the energy in the local rest frame at point $x$. The total particle yield of species $i$ is therefore

$$
N_{i}=\rho_{i}\left(T, \mu_{i}\right) \int_{\Sigma} d^{3} \sigma_{\mu}(x) u^{\mu}(x)=\rho_{i}\left(T, \mu_{i}\right) V_{\Sigma}\left(u^{\mu}\right)
$$

where only the total comoving volume $V_{\Sigma}$ of the freeze-out hypersurface $\Sigma$ depends on the flow profile $u^{\mu}$. Thus the flow pattern drops out from ratios of $4 \pi$ yields which therefore depend only on $T$ and the chemical potentials. These considerations are easily generalized to "fuzzy freeze-out" (i.e. freeze-out from a space-time volume rather than from a sharp hypersurface): as long as $T$ and $\mu_{i}$ are the same everywhere, $4 \pi$ particle ratios are independent of the collective dynamics of the source.

For heavy-ion collisions at SPS energies and below one should therefore perform a thermal analysis on $4 \pi$-integrated yields and not on particle ratios inside small rapidity windows. This requires a strong experimental effort to measure the rapidity distributions of as many particle species as possible over the full rapidity range.

\subsection{Non-constant thermodynamic parameters at freeze-out}

The second, even more serious problem is the observation that in reality freeze-out does not happen at constant temperature and chemical potential. For example, it was shown in Ref. 33 that a successful thermal description of the rapidity distributions of hadrons created in $200 A \mathrm{GeV} \mathrm{S}+\mathrm{S}$ collisions (in particular the different shapes of the rapidity distributions for $\Lambda$ and $\bar{\Lambda}, K^{+}$and $K^{-}$) requires not only strong longitudinal flow, but also a baryon chemical potential $\mu_{i}(\eta)$ which depends on the longitudinal position in

the fireball: the central rapidity region is baryon-poorer than the target and projectile fragmentation regions. A second example demonstrating this type of problem is the observed rapidity dependence of the $p_{\perp}$-slopes of the $h^{-}$and proton spectra [34] and of the $K_{\perp}$-slopes of the transverse HBT radius $R_{\perp}$ [24, 35]. According to a simultaneous analysis (as discussed in section 3.2 above) of spectra and correlations from $\mathrm{Pb}+\mathrm{Pb}$ collisions at the SPS by Schönfelder [35] the decrease of the inverse slope parameters away from midrapidity must be attributed to both a reduction of the transverse collective 
flow and of the freeze-out temperature $T(\eta)$. If true, this would speak against a constant freeze-out temperature.

In such a situation the thermal fit replaces the functions $T(\eta)$ and $\mu_{i}(\eta)$ by suitably averaged values $\bar{T}, \bar{\mu}_{i}$ (see Ref. [32] for a recent detailed study). Obviously the fit will then not be perfect: particle yields from a system in perfect local thermodynamic equilibrium (as, e.g., assumed in all hydrodynamic simulations), but with spatially varying temperature and chemical potentials, cannot be exactly recovered by a fit with constant temperature and chemical potential. In practice, this does not appear to be a very serious problem if one believes that the results from a recent thermal model analysis of particle yields from a hydrodynamic simulation [32] are representative for realistic situations: the freeze-out temperature reconstructed from the fit nearly coincided with the input temperature at which the hydrodynamic evolution was stopped, and the fitted chemical potentials agreed approximately with their average values across the freezeout surface. Nevertheless, small differences remain between the real yields from the hydrodynamic simulation and the yields returned by the thermal model fit. In a least mean square fit these systematic deviations would lead to a value for $\chi^{2} /$ d.o.f. which increases above all limits as the statistical error of the simulated ("measured") yields is further and further reduced, even though the system was, by construction, in perfect local thermal equilibrium.

This illustrates that global thermal fits to heavy-ion data can never be fully successful, due to the dynamics of the collision and its intricate influence on the freezeout process. For this reason one must not expect too much from the thermal model a perfect fit with extremely small $\chi^{2} /$ d.o.f. is not necessarily a good and often rather a bad sign, indicating accidental error correlations, e.g. due to the use of redundant fit parameters.

This leaves us with the question where to draw the line between "good" and "bad" thermal model fits, between success and failure of a thermodynamic description of relativistic heavy-ion collisions. The above discussion should have made it clear that $\chi^{2} /$ d.o.f. is not a good criterium for answering this question. On the other hand, a fit which reproduces particle yields which cover a range of more than three orders of magnitude with individual deviations of less than $\pm 25 \%$ [2] is obviously not bad. Quantitative model studies like those presented in [32 give us guidance for separating the grain from the straw; when supplemented by a thermal analysis of microscopic kinetic simulations as those presented at this meeting by Larissa Bravina [36], they are the foundations which we can use when collecting arguments in favor or against the formation of thermalized hot hadronic matter and quark-gluon plasma.

\section{Conclusions}

Let me summarize shortly: a thermal + flow analysis of yields, spectra and 2-particle correlations in $\mathrm{S}+A$ and $\mathrm{Pb}+\mathrm{Pb}$ collisions at the CERN SPS suggests

- the formation of a prehadronic state in which twice as much strangeness is produced 
as in $p p$ and $p A$ collisions and in which quarks are uncorrelated, i.e. they are not bound into color singlets;

- statistical hadronization of this state at $T_{\text {had }} \approx 170 \pm 20 \mathrm{MeV}$ with hadron abundances controlled by the Maximum Entropy Principle;

- rapid decoupling of the particle abundances, with chemical freeze-out temperatures $T_{\text {chem }} \approx T_{\text {had }}$ in sulphur-induced and $T_{\text {chem }} \leq T_{\text {had }}$ in $\mathrm{Pb}+\mathrm{Pb}$ collisions;

- elastic rescattering among the hadrons (dominated by $s$-channel resonances) after hadronization from the prehadronic state which leads to a cooling of the momentum spectra and the generation of (more) collective flow;

- finally, thermal freeze-out at $T_{\text {therm }} \approx 140-150 \mathrm{MeV}$ in $\mathrm{S}+A$ and at $T_{\text {therm }} \approx$ $120 \pm 10 \mathrm{MeV}$ in $\mathrm{Pb}+\mathrm{Pb}$ collisions, with average transverse collective flow velocities of order $\left\langle v_{\perp}\right\rangle \approx 0.4-0.5 c$.

Smaller collision systems distinguish themselves from larger ones not primarily by the achieved maximal energy density, but by the occupied collision volume in space and time. Compared to $\mathrm{S}+\mathrm{S}$ collisions, $\mathrm{Pb}+\mathrm{Pb}$ collisions live longer until thermal freeze-out, expand more in the tranverse direction, develop more transverse collective flow and cool down to lower (chemical and thermal) freeze-out temperatures. Of course, this view disagrees with the (present) majority opinion (I refer to the respective contributions to the Proccedings of "Quark Matter '97" [37) that the critical energy density for deconfinement can be crossed at fixed beam energy of $160 A \mathrm{GeV}$ by changing the size of the projectile and target or the collision centrality, and that the "anomalous" $J / \psi$ suppression observed in central $\mathrm{Pb}+\mathrm{Pb}$ collisions as a function of produced transverse energy [37, 38] signals this transition.

I find this interpretation irreconcilable with the systematics of light hadron production as discussed in this talk; a more consistent interpretation rests on the observation [38 that, as one increases $N_{\text {part }}$, one first sees "anomalous" suppression of the weakly bound $\psi^{\prime}$, then (in semiperipheral $\mathrm{Pb}+\mathrm{Pb}$ collisions) the "anomalous" suppression of the more strongly bound $\chi_{c}$ states (indirectly, via the disappearance of their $32 \%$ feed-down contribution to the measured $J / \psi$ yield), and only in very central $\mathrm{Pb}+\mathrm{Pb}$ collisions the disappearance of directly produced $J / \psi$ 's which are very strongly bound (this last part of the supression pattern still remains to be confirmed by an improved measurement at very high $E_{T}$ ). This suggests to me that what NA50 is seeing is not the onset of deconfinement (the latter is there even in $\mathrm{S}+A$ collisions), but the dissociation of more and more strongly bound heavy quark states (respectively the removal of the corresponding components in the $c \bar{c}$ wavefunction) by collisions with the dense partonic medium in the early stages of the collision. For the more strongly bound states most of these collisions will be subthreshold; for this reason a longer lifetime of the dense early stage, which is achieved in larger collision systems or more central collisions, is crucial for an efficient destruction not only of the weakly bound $\psi^{\prime}$, but also of the more strongly bound $\chi_{c}$ and $J / \psi$. Charmonium suppression is thus, in my opinion, 
more a lifetime effect than a deconfinement signal (although the necessary high density of scatterers with sufficiently large cross sections probably requires deconfinement, too).

The picture which I have painted is, I believe, intrinsically consistent. It is sufficiently simple to be attractive but also sufficiently sophisticated not to be unrealistic. It may not be unique, not least because of the intrinsic systematic uncertainties associated with thermal model analyses which I pointed out and not all of which are quantitatively understood. What is urgently needed is more high-quality data on the chemistry of $\mathrm{Pb}+\mathrm{Pb}$ collisions, the reconciliation of some puzzling discrepancies between different experiments as discussed at this meeting, and an improved systematics of the impact parameter and $A$-dependences, both in the light and heavy hadron sector.

\section{Acknowledgments}

The author acknowledges the hospitality at the INT (Seattle) in March/April 1998 where discussions with many colleagues allowed him to sharpen the arguments presented here. This work was supported by BMBF, DFG and GSI.

\section{References}

[1] Becattini F 1996 Z. Phys. C 69 485; Becattini F 1999 J. Phys. G: Nucl. Phys. 25, in press.

[2] Becattini F and Heinz U 1997 Z. Phys. C 76269

[3] Sollfrank J, Koch P and Heinz U 1991 Z. Phys. C 52593

[4] Schnedermann E, Sollfrank J and Heinz U 1993 Particle Production in Highly Excited Matter NATO ASI Series B303 (New York: Plenum) p 175

[5] For a recent compilation of theoretical analyses see Sollfrank J 1997 J. Phys. G: Nucl. Phys. 23 1903, especially figure 5 in the electronic preprint nucl-th/9707020 which was not included in the published version.

[6] Heinz U 1998 Nucl. Phys. A 638 357c

[7] Braun-Munzinger P and Stachel J 1998 Nucl. Phys. A 638 3c

[8] Metag V 1998 Nucl. Phys. A 638 45c

[9] Cleymans J and Redlich K 1998 Phys. Rev. Lett. 815284

[10] Becattini F, Gaździcki M and Sollfrank J 1998 Eur. Phys. J. C 5143

[11] Gaździcki M 1996 APH N.S.: Heavy Ion Physics 4 33, and references therein

[12] Andersen E et al (WA97 Coll.) 1998 Phys. Lett. B 433209 Králik I et al (WA97 Coll.) 1998 Nucl. Phys. A 638 115c Holme H K et al (WA97 Coll.) 1997 J. Phys. G: Nucl. Phys. 231851

[13] Lietava R et al (WA97 Coll.) 1999 J. Phys. G: Nucl. Phys. 25, in press; Caliandro R et al (WA97 Coll.) 1999 J. Phys. G: Nucl. Phys. 25, in press

[14] Evans D et al (WA85/WA94 Coll.) 1999 J. Phys. G: Nucl. Phys. 25, in press.

[15] Sollfrank J, Becattini F, Redlich K and Satz H 1998 Nucl. Phys. A 638 399c

[16] Sollfrank J 1998 private communication

[17] Rafelski J 1991 Phys. Lett. B 262333

[18] Bialas A 1998 Phys. Lett. B in press (hep-ph/9808434)

[19] Letessier J and Rafelski J 1998 hep-ph/9807346

[20] Sollfrank J, Heinz U, Sorge H and Xu N 1999 J. Phys. G: Nucl. Phys. 25, in press

[21] van Hecke H, Sorge H and Xu N 1998 Phys. Rev. Lett. 815764

[22] Xu N et al (NA44 Coll.) 1996 Nucl. Phys. A 610 175c

[23] Heinz U 1996 Nucl. Phys. A 610 264c and references therein 
[24] Appelshäuser H et al (NA49 Coll.) 1998 Eur. Phys. J. C 2661

[25] Wiedemann U A, Tomášik B and Heinz U 1998 Nucl. Phys. A 638 475c

[26] Kämpfer B et al 1997 J. Phys. G: Nucl. Phys. 232001

[27] Schnedermann E, Sollfrank J and Heinz U 1993 Phys. Rev. C 482462 Schnedermann E and Heinz U 1994 Phys. Rev. C 501675

[28] Heinz U 1998 in: Relativistic Aspects of Nuclear Physics (Proceedings of the Fifth Rio de Janeiro International Workshop, T. Kodama et al , eds.) (Singapore: World Scientific) p 19

[29] Appelshäuser H et al (NA49 Coll.) 1998 Phys. Rev. Lett. 804136

[30] Poskanzer A and Sorge H 1998 private communications and work in preparation

[31] Mermin D 2000 Physics Today 52 to be published

[32] Sollfrank J 1998 Preprint nucl-th/9811078

[33] Slotta C, Sollfrank J and Heinz U 1995 Strangeness in Hadronic Matter, AIP Conference Proceedings 340 (Woodbury: AIP Press) p 462

[34] Jacobs P 1997 Proceedings of ICPA-QGP97, Jaipur, India, March 17-21, 1997, NA49 note number 123

[35] Schönfelder S 1997 PhD thesis, MPI für Physik, München, NA49 note number 143

[36] Bravina L V et al 1999 J. Phys. G: Nucl. Phys. 25, in press

[37] Hatsuda T et al (eds.) 1998 Nucl. Phys. A 638

[38] Abreu M C et al (NA50 Coll.) 1997 Phys. Lett. B 410337

Romana A et al (NA50 Coll.) 1998 XXXIIIrd Rencontres de Moriond, 21-28 March 1998, Les Arcs, France 